\title{
Anger-based BCI Using fNIRS Neurofeedback
}

\author{
Gabor Aranyi \\ Teesside University \\ School of Computing \\ Middlesbrough, TS1 3BA, UK \\ g.aranyi@tees.ac.uk
}

\author{
Fred Charles \\ Teesside University \\ School of Computing \\ Middlesbrough, TS1 3BA, UK \\ f.charles@tees.ac.uk
}

\author{
Marc Cavazza \\ Teesside University \\ School of Computing \\ Middlesbrough, TS1 3BA, UK \\ m.o.cavazza@tees.ac.uk
}

\begin{abstract}
Functional near-infrared spectroscopy (fNIRS) holds increasing potential for Brain-Computer Interfaces (BCI) due to its portability, ease of application, robustness to movement artifacts, and relatively low cost. The use of fNIRS to support the development of affective BCI has received comparatively less attention, despite the role played by the prefrontal cortex in affective control, and the appropriateness of fNIRS to measure prefrontal activity. We present an active, fNIRS-based neurofeedback (NF) interface, which uses differential changes in oxygenation between the left and right sides of the dorsolateral prefrontal cortex to operationalize BCI input. The system is activated by users generating a state of anger, which has been previously linked to increased left prefrontal asymmetry. We have incorporated this NF interface into an experimental platform adapted from a virtual 3D narrative, in which users can express anger at a virtual character perceived as evil, causing the character to disappear progressively. Eleven subjects used the system and were able to successfully perform NF despite minimal training. Extensive analysis confirms that success was associated with the intent to express anger. This has positive implications for the design of affective BCI based on prefrontal asymmetry.
\end{abstract}

\section{Author Keywords}

Brain-Computer Interface; Approach Motivation; Neurofeedback; Functional Near-Infrared Spectroscopy.

\section{ACM Classification Keywords}

H.5.2. Information interfaces and presentation: User Interfaces.

\section{INTRODUCTION}

The rapid progress of fNIRS applications to user interfaces makes it possible to envision a new generation of BrainComputer Interfaces (BCI), with improved spatial

Permission to make digital or hard copies of all or part of this work for personal or classroom use is granted without fee provided that copies are not made or distributed for profit or commercial advantage and that copies bear this notice and the full citation on the first page. Copyrights for components of this work owned by others than the author(s) must be honored. Abstracting with credit is permitted. To copy otherwise, or republish, to post on servers or to redistribute to lists, requires prior specific permission and/or a fee. Request permissions from Permissions@acm.org.

UIST'15, November 08 - 11, 2015, Charlotte, NC, USA

Copyright is held by the owner/author(s). Publication rights licensed to

ACM.

ACM 978-1-4503-3779-3/15/11 ..\$15.00

DOI: http://dx.doi.org/10.1145/2807442.2807447 resolution, better signal stability and greater robustness to motion artefacts $[1,40]$. One particular interest for the development of BCI is the accessibility of specific prefrontal areas to fNIRS [11, 29, 43]. Ayaz et al. [5] have explicitly mentioned accessing signals from the dorsolateral prefrontal cortex (DL-PFC) in their application. This has important implications, as several anatomical areas of the DL-PFC are involved in executive functions [5] and emotional regulation $[11,22]$.

This could help addressing a persistent challenge for the design of affective BCI, which is to relate emotional processes to specific brain regions whose activity is accessible to measurement [19]. Prefrontal asymmetry is considered an important marker of affective-motivational state, in particular the approach/withdrawal ${ }^{1}$ dimension [10], and has received considerable attention in clinical neuroscience. Its potential in the development of affective BCI has been recognized $[28,44]$ and has been the object of recent experiments [9], which exploited the original finding that asymmetry could be controlled by subjects through neurofeedback (NF) in clinical applications [35].

Zotev et al. [45] have conducted simultaneous EEG/fMRI experiments that confirmed prefrontal asymmetry as the anatomical substrate for approach and its variations during NF. Since fNIRS captures metabolic patterns similar to those observed in fMRI, this was a further encouragement to explore the use of fNIRS for prefrontal asymmetry NF.

One of the limitations of previous attempts at using prefrontal asymmetry as a measure of approach in BCI is the non-specific nature of its activation. Because approach is connected to appetitive stimuli [7, 34], it is not clearly dissociated from valence, and the expression of positive thoughts has been reported to induce prefrontal asymmetry. This was also described in Zotev's NF experiments [45], where the recollection of pleasant autobiographical memories was reported to induce prefrontal asymmetry. We observed similar phenomena in our previous EEG-based work on empathic BCI [12], where subjects' attitudes were split between expressing empathy and generic positive

1 Approach is related to the energization of behavior towards (positive) stimuli, whereas withdrawal (or avoidance) is related to directing behavior away from (negative) stimuli [10]. 


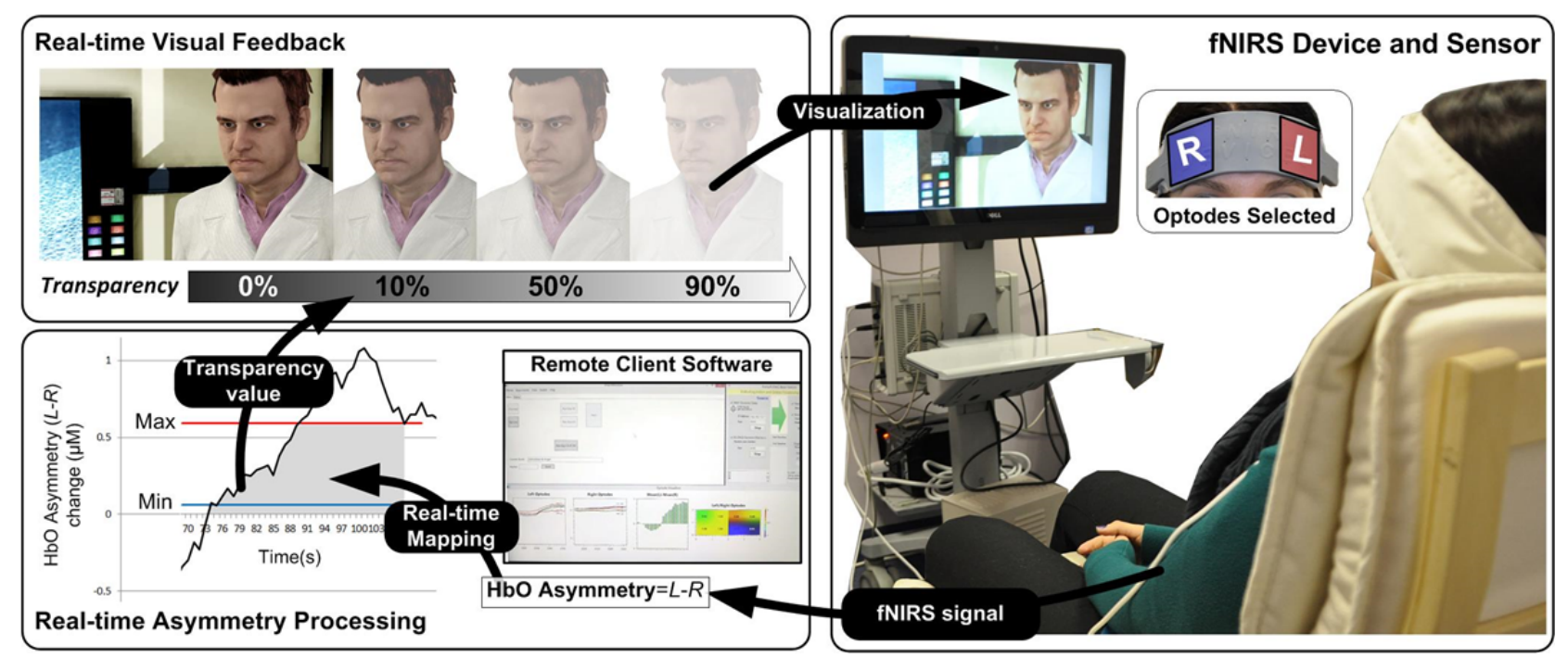

Figure 1. System Architecture. Input fNIRS signal is sampled at $2 \mathrm{~Hz}$ and supports the calculation of a frontal asymmetry score. During NF, that score is linearly mapped to the visual appearance of a virtual character to reflect the expression of anger by the user; higher levels of prefrontal asymmetry result in the progressive disappearance of the character.

thoughts, sometimes autobiographical as well, hence unrelated to the visual NF target. However, for the future development of BCI based on approach/withdrawal, it is essential to establish that a specific approach signal can be produced independently of valence. Prefrontal asymmetry can be induced by positive thoughts only, some containing an approach component; however, Harmon-Jones [15] has established that in the case of anger, approach is clearly dissociated from valence: an anger response being characterized by increased left prefrontal asymmetry associated to negative valence (i.e. anger represents approach towards negatively perceived stimuli). A BCI strategy based primarily on anger would confirm the usability of approach for the design of affective BCI systems. In this paper, we describe such a system, in which users direct their anger at a virtual character.

\section{PREVIOUS AND RELATED WORK}

A significant fraction of previous fNIRS work has been dedicated to passive acquisition of user cognitive states [40], which has been used to assess cognitive workload [18], information presentation [32], or level of difficulty [13]. This has been applied to usability studies [17] and the development of adaptive interfaces [14], for instance through dynamic difficulty adjustment [2], recommender systems [31], or new pointing techniques [3].

However, our interest is instead in the use of fNIRS for active BCI, which has been introduced by Ayaz et al. [6] in the form of an fNIRS NF interface. The task given to users was to increase a bar value up to a certain predefined threshold: crossing the threshold defined NF success, and users' performance was further measured as the time required to cross the threshold. This design was later extended and incorporated to a more realistic environment in the form of a 3D game [5]. Oum et al. [30] developed MindTactics, a 3D game environment incorporating fNIRSbased BCI, to experiment with BCI-based gameplay as well as supporting $\mathrm{BCI}$ research. User interaction is based on maintaining concentration, which is reflected through display bars, despite various distractors in the 3D environments.

The potential use of prefrontal asymmetry to support affective BCI has been discussed in various reviews [28], although without reporting specific implementations. Wehbe et al. [44] reported the use of EEG prefrontal asymmetry as a measure of arousal rather than approach, and applied this to user experience during game-based learning [44]. Karran et al. [21] explored the role of the PFC in subjects' aesthetic experiences using prefrontal asymmetry as a measure of valence. In our previous work [12], we explored the use of EEG alpha prefrontal asymmetry NF to implement affective BCI input to an interactive narrative. In this system, users would express positive empathy towards a $3 \mathrm{D}$ virtual character, resulting in a more favorable evolution of the story. Data acquired from simultaneous fMRI analysis of the PFC was consistent with the original hypothesis but could not be interpreted further, in view of the small number of subjects.

\section{SYSTEM OVERVIEW}

We designed a NF system around commercial fNIRS equipment. The overall architecture of the system is presented on Figure 1. Our system computes real-time variations of prefrontal asymmetry from the acquisition of hemodynamic data and produces visual feedback to the user, based on the rendering and animation features of a 3D game engine $\left(\mathrm{UDK}^{\mathrm{TM}}\right)$. 


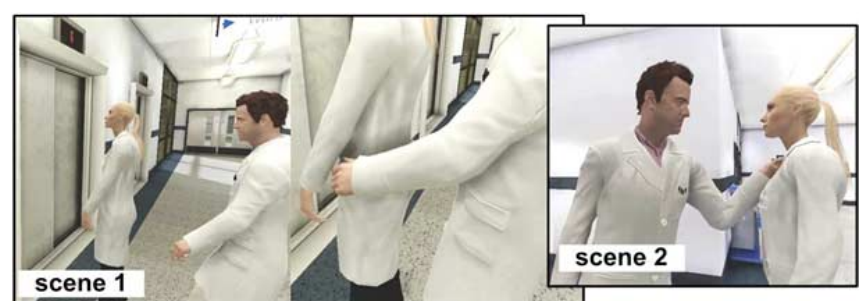

Figure 2. User attitudes towards the target character are prompted by showing user excerpts from the background narrative, portraying him as mischievous.

The scenario for the anger-based interface consists for the user to express anger against a virtual character. Throughout specific NF epochs, users actively express anger towards the character: in return, based on their variation in prefrontal asymmetry, the character's images fades from the environment, as if they had the ability to erase him. Like with most NF systems, the core calculation consists in the real-time mapping between the neural signal (here prefrontal asymmetry) and the visual feedback signal (image transparency for the virtual character).

We developed experimental software which automates protocol timing, data collection, stimulus presentation, as well as embedding the NF module. Remote client software connected to the data collection software manages the realtime processing of values of oxygenated hemoglobin $(\mathrm{HbO})$ concentration changes, the triggering of baseline capture, as well as sending marker information for post-processing of fNIRS data. The metric of inter-hemispheric difference in the level of $\mathrm{HbO}$ change is calculated in real-time as the difference of the four leftmost and four rightmost channels (i.e., asymmetry $=L-R,[11]$ ). The graphical user interface (Figure 1), developed using $\mathrm{C \#}$ and Windows WPF, includes a real-time visualization of $\mathrm{HbO}$ changes and asymmetry values. All these variables are logged during the experiment to facilitate the post-processing of the collected data. This graphical user interface was also used to manage the running of the experiment itself, which included displaying the required epochs (text, image, video, or more complex visualization) and managing their duration and the synchronization of all the software components. Finally, this interface was also used to implement visual feedback.

In the next sections, we describe our experimental design and give details on the real-time aspects of NF implementation; we also present global success criteria for a neurofeedback epoch. We then analyze experimental results to evaluate $\mathrm{BCI}$ performance.

\section{METHODS}

\section{Subjects}

Twelve adults were recruited at the authors' institution. One subject had to be excluded due to technical problems, which left an effective sample size of 11 (mean age $=33.55$ years, $\mathrm{SD}=11.53$, range: [24; 59], 5 female). Subjects had no history of psychiatric conditions and were right-handed.
They all provided written consent prior to participation. The experiment was approved by a research ethics committee at the authors' institution. Each subject was compensated with an online retailer voucher equivalent to $\$ 30$.

\section{Apparatus}

We used an fNIR Optical Brain Imaging System (fNIR400) by Biopac Systems for data acquisition. Raw fNIRS data and oxygenation values were collected with $2 \mathrm{~Hz}$ sampling rate (using COBI Studio and FnirSoft v3.5), and was sent to the experimental software over TCP/IP (using FnirSoft DAQ Tools). A 16-channel sensor with a fixed $2.5 \mathrm{~cm}$ source-detector separation was placed on the subjects' forehead. For real-time application we used measurements of changes in $\mathrm{HbO}$ concentration, as opposed to deoxygenated or total hemoglobin ( $\mathrm{HbR}$ and $\mathrm{HbT}$, respectively), because it has been reported to be sensitive to task-related activation in the PFC [3] and to affect-related experimental manipulation in the DL-PFC [43], and it has been successfully applied in research involving approach/withdrawal-related manipulation [27]. Values of $\mathrm{HbO}$ concentration changes were averaged over four leftmost and four rightmost channels ${ }^{2}$ (located over the left and right DL-PFC, respectively) to derive a simple metric of inter-hemispheric difference in the level of $\mathrm{HbO}$ change (see [11]) that could account for left prefrontal asymmetry.

We followed the recommendations of Solovey et al. [39] regarding the use of fNIRS in a HCI setting. Subjects were seated approximately 47 " $(120 \mathrm{~cm})$ from a 24 " flat monitor in a dimly-lit, quiet (but not soundproof) room in a comfortable chair to minimize movements, with the fNIRS probe positioned over their forehead and covered with nontransparent fabric to prevent ambient light reaching the sensors (see Figure 1). Subjects were instructed to refrain from moving their limbs, frowning and talking during datacollection blocks indicated by the experimental software.

\section{Subjects Instructions and Questionnaires}

To set a context for the NF task involving anger expression, subjects watched $3 \mathrm{D}$ animations from a narrative set in a hospital environment, comprising four short scenes (overall length: 71s) illustrated in Figure 2. The objective of these scenes was to introduce the target character as a despicable subject, one at which it would be appropriate to target anger. The background narrative is a medical drama, in which the character features as unethical and as a bully. In the first scene, he is shown sexually harassing a female colleague. In other scenes, he belittles colleagues and ridicules their professional abilities, or he boasts about getting away with professional malpractice. To assess the effectiveness of setting the context, we asked subjects to

\footnotetext{
${ }^{2}$ See [33] for a similar approach to combining specific channels to form a single measure.
} 


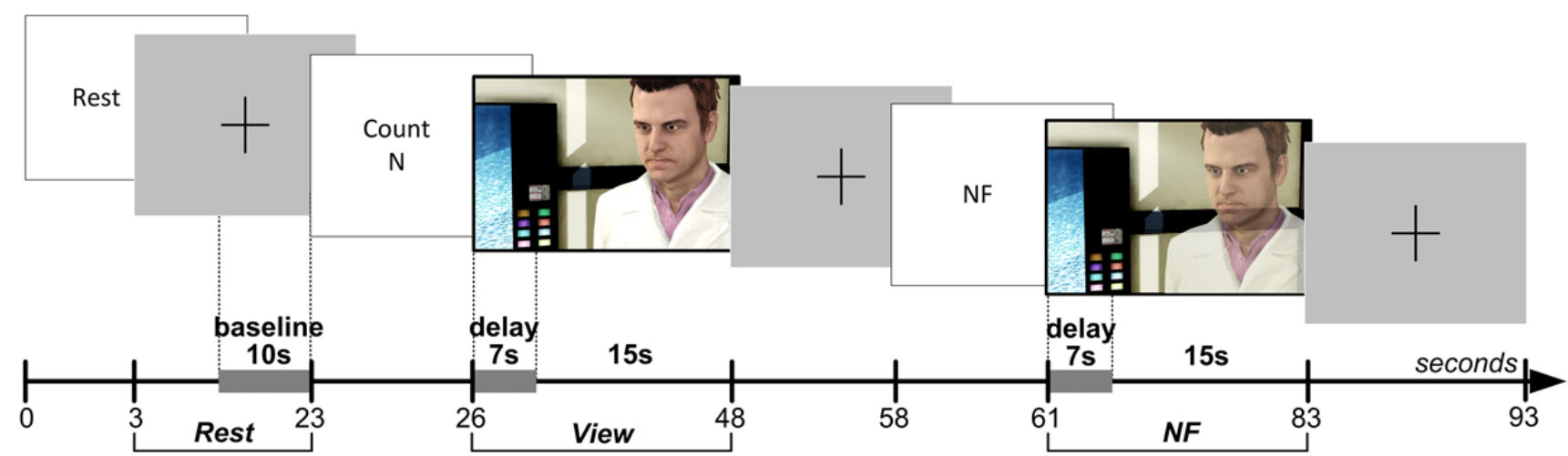

Figure 3. Block Design. The main three epochs of the block are a Rest epoch, a View epoch, and an NF epoch (all 3 epochs were preceded by a $3 \mathrm{~s}$ prompt to count). View epochs are used to provide a reference for the following NF epoch, also controlling for visual stimulus to avoid artifacts due to spontaneous affective response.

rate the seriousness of the presented case of bullying on a 1 to 10 scale. The smallest seriousness rating was 7 $(\mathrm{M}=8.36, \mathrm{SD}=0.95$, range: $[7 ; 10])$, indicating that the clip set an adequate context for expressing anger towards the target character (i.e. the male doctor or 'the bully').

Since the experiment involved expressing anger towards a virtual agent, we included in the protocol the psychometric measurement of anger using the State-Trait Anger Expression Inventory (STAXI-2; [41]) to assess the relationship between anger disposition and NF success through the expression of anger. Subjects completed the 10item trait scale of STAXI-2 to assess their disposition to perceive certain situations as annoying or frustrating, and their tendency to respond to such situations with elevated state anger.

Next, subjects were instructed that they would go through a sequence of blocks, each comprising three main epochs: Rest, View and NF (see Figure 3). During Rest, the baseline for calculating $\mathrm{HbO}$ data is acquired for the block as a whole. The View epochs correspond to control conditions, during which the subjects watch an idle animation of the character while given a cognitive task (counting, see [29]) that keeps them in a neutral state, hence providing a reference for prefrontal asymmetry levels. Finally, the $N F$ epochs consist in subjects expressing anger towards the character and receiving visual feedback. Subjects were instructed to express their anger "mentally" and did not receive specific instructions that could bias their cognitive strategy. They were simply reminded that their anger had to be targeted at the character.

Each subject completed two training blocks to get acquainted to the task, followed by six blocks for the experiment itself, during which $\mathrm{HbO}$ asymmetry was monitored and recorded. After each block, subjects rated the amount of anger they felt they managed to express towards the target character on a 1 to 10 scale. They were then also asked to describe their cognitive strategy in expressing anger during the block.

\section{Block Structure: View and Neurofeedback Epochs}

Each block started with a verbal prompt to rest (lasting 3s) presented in the center of a grey screen. The prompt was replaced by a crosshair that remained on screen for $20 \mathrm{~s}$. The subject was instructed to keep looking at the center of the screen and relax. The last 10s of the Rest epoch (20 observations sampled at $2 \mathrm{~Hz}$ ) was used to calculate the baseline for the block as a whole (see [36]).

The View epoch started with a written prompt to count and a single-digit integer. The task was to count backwards from 500 by subtracting the provided integer. After $3 \mathrm{~s}$, the prompt was replaced with an idle video of the target character (the "bully") that remained on screen for 22s. The subject was instructed to keep looking at the screen and carry on doing the counting task in her head until the character was replaced by a crosshair. It was followed by a 10 s between-epoch interval, during which the crosshair was displayed again on a gray background, and the subject was instructed to abandon the counting task and relax.

The $N F$ epoch started with a written prompt to express anger. After $3 \mathrm{~s}$, the prompt was replaced by a still shot from the idle video the subject was previously shown during View; this picture was used as the feedback channel. The subject was instructed to keep looking at the screen while expressing her anger mentally towards the target character, and that she can make the picture of the character fade away from the surroundings if she expressed anger effectively. The scene was replaced by the crosshair after 22s. No behavioral expression of anger (such as marked facial expressions) was observed for the vast majority of users during experiments. Each block was finished with the crosshair presented at the center of a grey screen for 10s. 

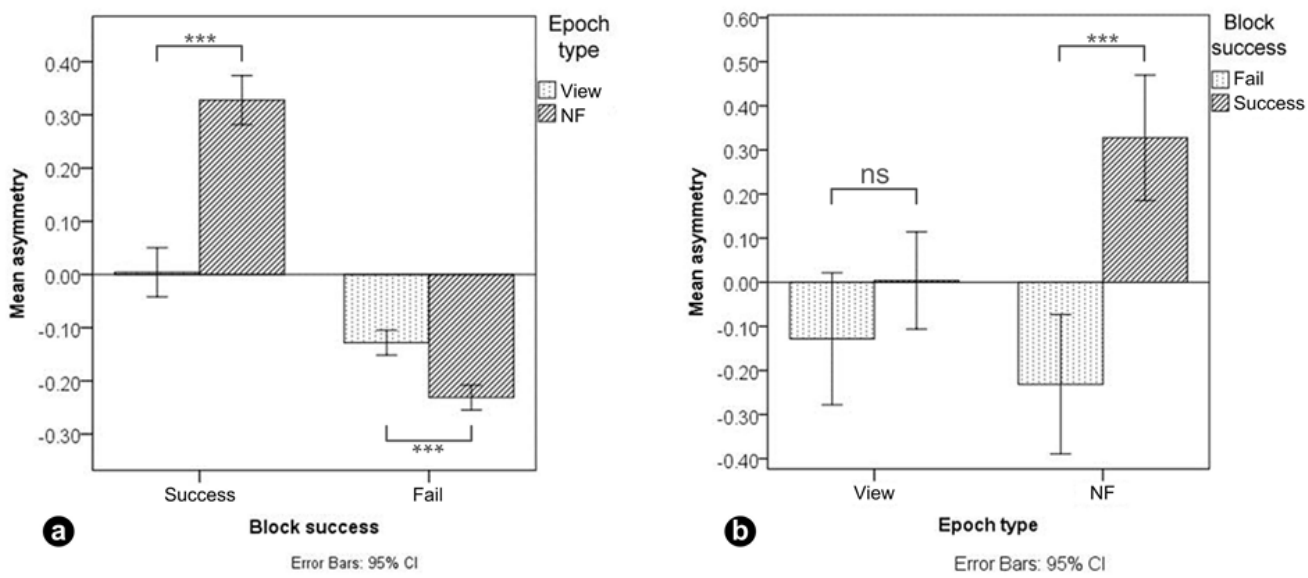

Figure 4: Differences in asymmetry in successive View and NF epochs. (a) Comparisons across epoch type (within-subject factor; note that the error bars are based on within-block variance between successive View and NF epochs). (b) Comparisons across block success (between-subject factor): asymmetry in View epochs did not differ significantly across levels of block success, confirming that success was primarily due to an increase in left-side activation during $N F$ blocks.

It should be noted that, rather than inducing anger in subjects through experimental manipulation (e.g., [16]), we asked them to generate a state of anger towards a target. This was for the purpose of using anger as voluntary affective input through fNIRS, rather than attempting to detect induced anger with this method.

\section{Neurofeedback Success Criteria}

Although subjects get an indication of success through visual feedback, there is a need to objectively assess success over an entire block, as well as quantifying it for further analysis. fNIRS signals are relative to a baseline [4] and it can be difficult to compare them across subjects [37]. Moreover, the magnitude of oxygenation changes can also differ substantially across blocks within the same subject. Therefore, we designed our success criterion for defining and quantifying block success to mitigate the issue of comparability.

As discussed above, we used the last 10 seconds of the Rest epoch at the start of each block to calculate the baseline for the block [36]. Since we characterized asymmetry as the difference between average $\mathrm{HbO}$ changes in the left and right DL-PFC, asymmetry during baseline was defined as zero. This operationalization of asymmetry yielded intervallevel data (values had no meaningful absolute zero point). Consequently, we could not use a ratio of task/no-task signals for defining and quantifying success, and providing feedback (e.g., Sarkheil et al. [38]).

As a solution, we treated a block as successful if the mean of asymmetry values during the $N F$ epoch was statistically significantly larger than the mean of asymmetry values during the View epoch within the same block. As opposed to simply comparing asymmetry scores during $N F$ to the overall baseline, we compared asymmetry scores between the successive View and $N F$ epochs because the visual stimulus was very similar (and conceptually the same) in the two epochs. Moreover, we set conditions for controlling subjects' cognitive activity during these epochs (counting during View and expressing anger during $N F$ ), whereas thought processes during Rest were not controlled. Thus, the View epoch served as a control condition within each block.

Because the hemodynamic response measured by fNIRS occurs in approximately $7 \mathrm{~s}$ [8], we discarded the first $7 \mathrm{~s}$ of data in each View and $N F$ epoch for determining block success. The system determined block success by performing and independent t-test on the set of asymmetry scores collected during successive View and $N F$ epochs within a block. In particular, it calculated mean and standard deviation (SD) of asymmetry scores in both epochs, and then calculated the $t$ value. Since removing the first 7s left $15 \mathrm{~s}$ of data per epoch (at least 29 data points sampled at $2 \mathrm{~Hz}$ ), the software used the $\mathrm{t}$ critical value of 2.05 with 28 degrees of freedom for $p$ (two-tailed) $=.05$ as a threshold for success. Furthermore, to quantify the extent of block success by expressing the distance of the distribution of asymmetry scores during successive View and $N F$ epochs, the experimental software calculated the Cohen's d effect-size measure, which is the difference between two means divided by the pooled standard deviation. This way we characterized each block with a dichotomous success value (success/fail) and a continuous success score (Cohen's $d$ or $d$ for short) that reflects the distance between the distribution of asymmetry values between View and $N F$ epochs within the same block.

\section{Real-time Neurofeedback Mapping}

Mapping is an essential part of any NF software, as it ensures that the individual variations in target neural activity are consistently translated into variations of the feedback signal. In the present case, this involves determining the individual range of variation of $\mathrm{HbO}$ asymmetry and establishing a mapping function between 


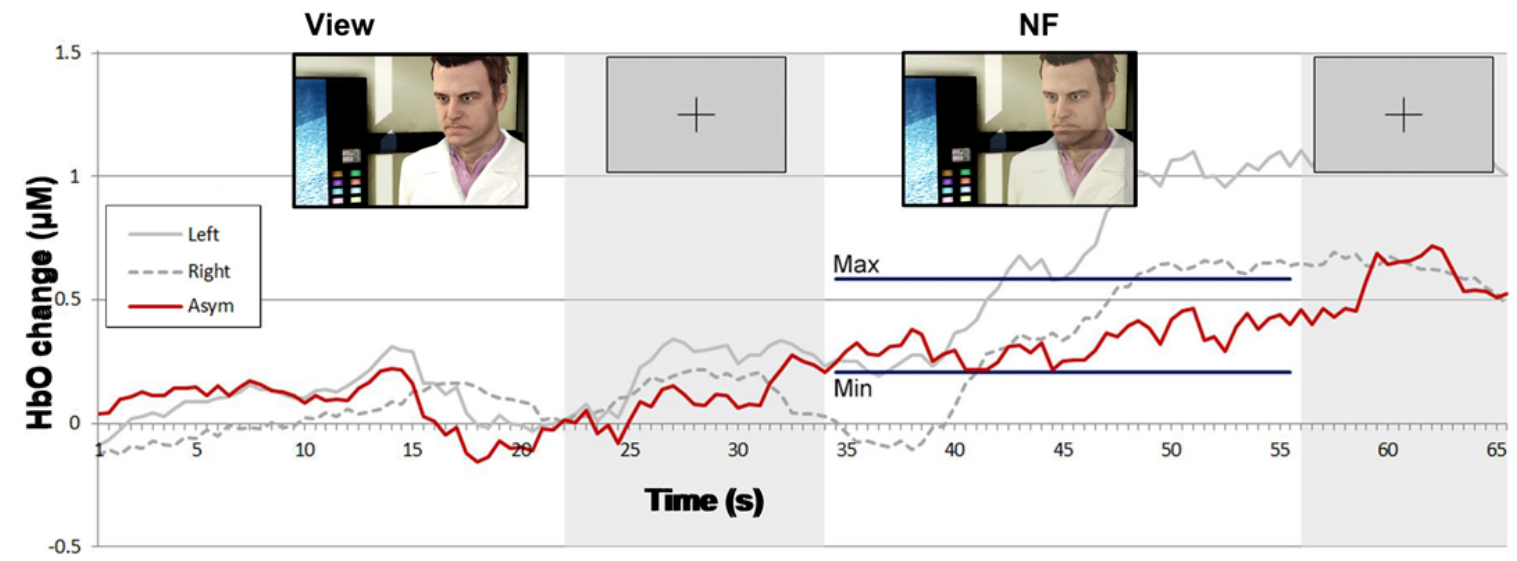

Figure 5. Example of asymmetry variation over time in a successful block. The horizontal lines in the $N F$ epoch (labelled Min and Max) represent the range of values which determines NF mapping. Note that during the $N F$ epoch, $\mathrm{HbO}$ increases bilaterally, with a marked asymmetry to the left.

asymmetry values in that range and the $0-100 \%$ variation of the visual signal (image transparency, see Figure 1). Within each block, we used the asymmetry scores during the View epoch to determine the range for mapping asymmetry values to the feedback channel during the $N F$ epoch. This was calculated by the system during the between-epoch interval, to automatically parameterize mapping during $N F$.

We defined the minimum point for mapping (Min) as the mean of asymmetry values during View plus 1.28 times their SD (the asymmetry value for which the visual feedback is equal to $10 \%$ transparency). Assuming normally distributed asymmetry scores, this threshold would result in no feedback for $90 \%$ of the spontaneous asymmetry variations during View. This approach to the determination of NF threshold for left-asymmetry is consistent with the original one of Rosenfeld et al. [35] for EEG. To determine the (Max) value of asymmetry, resulting in maximum visual feedback (i.e. $100 \%$ transparency), we increased the threshold for feedback (Min) by the maximum variation range of asymmetry during View.

The feedback range thus determined during the View epoch immediately preceding $N F$, mapping can take place in realtime between asymmetry values collected during $N F$ and the visual transparency of the target character over the background (Figure 1). The camera location and angle within the 3D scene were generated identical to the View epoch's visualization, and the target character's image was overlaid on top of the 3D scene background image. Asymmetry values within the range [Min; Max] were mapped linearly onto the visual transparency range of 0 $100 \%$ (Figure 1), with the same $2 \mathrm{~Hz}$ frequency as the acquisition of asymmetry values. For real-time updating, asymmetry values reaching the feedback threshold (Min) were mapped by $10 \%$ increments in transparency, while values reaching (and exceeding) the maximum (Max) were mapped to $100 \%$ transparency.

\section{RESULTS AND ANALYSIS}

\section{Block Success}

We defined block success as a statistically significant increase in average asymmetry during the $N F$ epoch compared to the View epoch within the same block. Out of the total 66 blocks collected from the 11 subjects, 38 were successful (58\%). Eight out of 11 subjects (73\%) completed at least half of the blocks successfully (Median $=4$ blocks out of 6$)^{3}$. One subject had no successful blocks; no subject completed all blocks successfully. We complemented the dichotomous success criterion with a continuous success score, characterized with the Cohen's d effect-size measure. Mean score in successful blocks was $\mathrm{d}=2.39(\mathrm{SD}=1.56)$, which corresponds to an average $23 \%$ overlap in asymmetry scores between View and $N F$ (assuming normally distributed asymmetry values). The smallest effect size we could detect (in $15 \mathrm{~s}$ of asymmetry data sampled at $2 \mathrm{~Hz}$, with a two-tailed $\mathrm{p}<.05$ criterion) was $\mathrm{d}=0.59$ (medium), which corresponds to a $76 \%$ overlap between asymmetry values during successive View and $N F$ epochs. This indicates that our success criterion was sensitive to detect relatively small increases in asymmetry, while asymmetry values in most successful blocks had small overlap between View and $N F$.

To determine if observed effects were indeed related to users' intentional behavior, subjects rated the amount of anger they expressed towards the target character during the $N F$ after each block on a 1 to 10 scale. In successful blocks, the self-reported magnitude of expressed anger was significantly correlated with the success score, $\mathrm{r}=.33$, $\mathrm{p}=.043$. Conversely, the magnitude of expressed anger was uncorrelated with the success score in failed blocks,

\footnotetext{
${ }^{3}$ See [9], and also [35] for a similar way of defining NF success at subject level.
} 


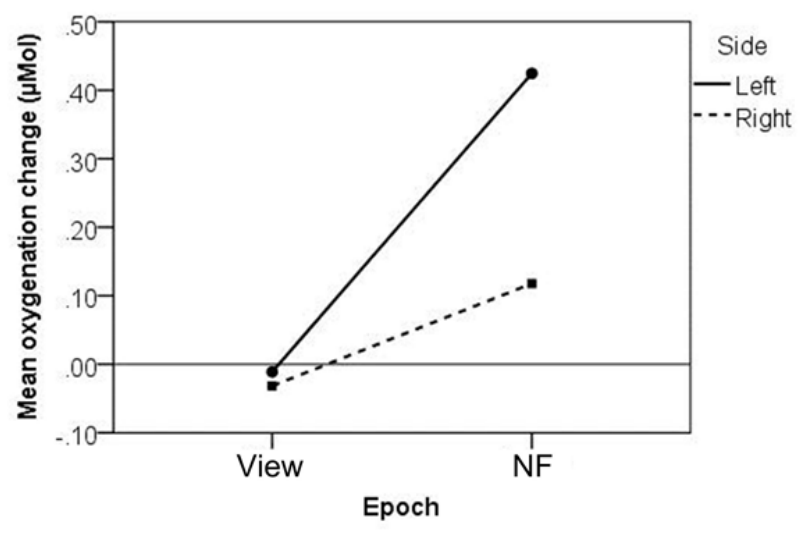

Figure 6: Differential increase in left-side oxygenation in successful blocks.

$\mathrm{r}=-.07, \mathrm{p}=.725, \mathrm{~ns}$. In addition, the self-reported magnitude of expressed anger during $N F$ was uncorrelated with STAXI-2 trait and state scales. However, the temperament subscale of the STAXI-2 trait scale was significantly correlated with the success score, $r=.27$, $\mathrm{p}=.026$, which suggests that people with higher dispositional trait anger may be more effective in upregulating frontal asymmetry by mentally expressing anger towards a target.

\section{Asymmetry across View and Neurofeedback Epochs}

Another validation of the interface consists in confirming that spontaneous fluctuations of asymmetry cannot account for the observed effects. To that effect, we explored changes in asymmetry between View and $N F$ epochs in relation to blocks success with a 2 × 2 Mixed ANOVA. Note that the significant interaction effect between success and epoch type $(\mathrm{F}(1,64)=56.78, \mathrm{p}<.001)$ is trivial, since we defined block success with significantly larger asymmetry during $N F$ than during View. We therefore concentrate on post-hoc pairwise comparisons (see Figure 4). In successful blocks, average asymmetry during View was zero $(\mathrm{M}=0.00, \mathrm{SD}=0.34)$, while average asymmetry during $N F(\mathrm{M}=0.33, \mathrm{SD}=0.43)$ was significantly larger, $\mathrm{t}(37)=7.10, \mathrm{p}<.001, \mathrm{r}=.76$ (large). Conversely, failed blocks where characterized with a decrease in asymmetry between View and NF, $\mathrm{t}(27)=-4.54, \mathrm{p}<.001, \mathrm{r}=.66$ (large).

Additionally, asymmetry during View did not differ significantly in successful $(\mathrm{M}=0.00, \mathrm{SD}=0.34)$ and failed $(\mathrm{M}=-0.13, \mathrm{SD}=0.39)$ blocks, $\mathrm{t}(64)=1.48, \mathrm{p}=.143, \mathrm{~ns}, \mathrm{r}$ $=.18$ (small), while asymmetry during $N F$ was significantly larger in successful blocks $(\mathrm{M}=0.33, \mathrm{SD}=0.43)$ than in non-successful ones $(\mathrm{M}=-0.21, \mathrm{SD}=0.41), \mathrm{t}(64)=5.31, \mathrm{p}$ $<.001, \mathrm{r}=.55$ (large). These findings indicate that successful and non-successful blocks differed predominantly on the basis of increase in asymmetry during $N F$, as opposed to lower levels of asymmetry during View. This confirms the role of active expression of anger over baseline fluctuations (Figure 5).

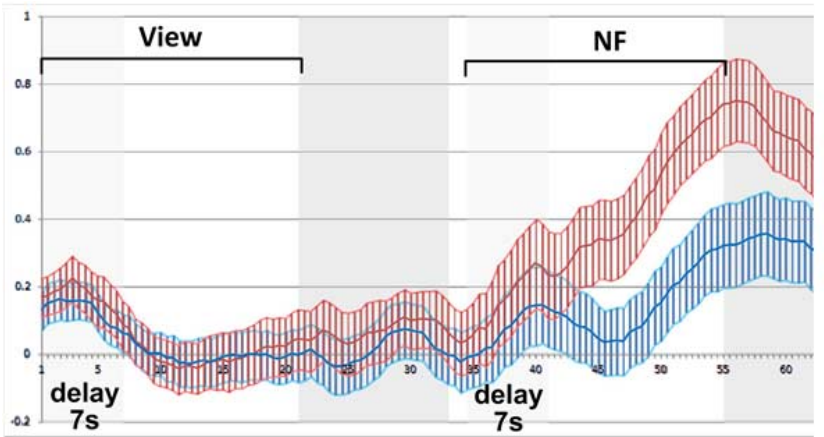

Figure 7. Mean and standard error of fNIRS signal (HbO) across all successful blocks $(n=38)$ for left $($ Red) and right (Blue) sides separately. Note that the signal overlaps on the two sides during View, while left rises above right during $N F$.

\section{Lateralized Difference in Activation across View and Neurofeedback Epochs}

To explore how oxygenation changes on both sides contributed to the increase in asymmetry from View to $N F$ within successful blocks, we conducted a 2 x 2 mixed ANOVA on average oxygenation changes with Epoch ( View/NF) as within-subject factor and Side (Left/Right) as between-subject factor. The analysis revealed a significant interaction, $\mathrm{F}(1,74)=7.86, \mathrm{p}=.006$, partial $\eta^{2}=.10$, which indicates that oxygenation changed differentially on the left and right sides between consecutive View and NF epochs (see Figure 6). Conducting the same analysis for failed blocks revealed no significant interaction, $\mathrm{F}(1,54)=0.16$, $\mathrm{p}=.688$, ns, which indicates no differential change in oxygenation from View to $N F$ in failed blocks. We conducted post-hoc pairwise comparisons to break down the significant interaction effect. In successful blocks (Figure 6), on the left side, average oxygenation increase from View $(\mathrm{M}=-0.01, \mathrm{SD}=0.44)$ to $N F(\mathrm{M}=0.42$, $\mathrm{SD}=0.61)$ was statistically significant, $\mathrm{t}(37)=6.20$, $\mathrm{p}<.001, \mathrm{r}=.71$ (large). On the right side, average oxygenation increase from View $(\mathrm{M}=-0.03, \mathrm{SD}=0.39)$ to $N F(\mathrm{M}=0.12, \mathrm{SD}=0.62)$ approached significance, $\mathrm{t}(37)=2.01, \mathrm{p}=.051, \mathrm{r}=.31$ (medium). Note that the magnitude and effect-size of oxygenation increase was markedly larger on the left side. These findings indicate that although oxygenation increased on both sides during $N F$ in successful blocks, asymmetry was predominantly a result of differential increase in left-side oxygenation (Figure 7). Conversely, post-hoc pairwise comparisons conducted on non-successful blocks revealed no significant change in oxygenation between View and NF on either side (Left: $\mathrm{t}(27)=0.39, \mathrm{p}=.703$; Right: $\mathrm{t}(27)=.98, \mathrm{p}=.335)$. Additionally, STAXI-2 trait anger was significantly correlated with the magnitude of left-asymmetry during $N F$, $\mathrm{r}=.33, \mathrm{p}<.01$, which is consistent with the findings of Harmon-Jones [15]. Specifically, the temperament subscale of trait anger was significantly and positively correlated with left-side activation increase during $N F$ epochs, $\mathrm{r}=.27$, 
$\mathrm{p}=.03$, while it was uncorrelated with right-side activation, $\mathrm{r}=.01, \mathrm{p}=.96$, ns.

\section{Cognitive Strategies for Expressing Anger}

For most users, the unusual experience of controlling brain areas activation through NF requires them to adopt a cognitive strategy, i.e., a set of thought contents on which to concentrate during NF epochs. Cognitive strategies are known to constitute an important aspect of NF systems [25], including those based on fNIRS [5]. After completing each block, subjects were thus asked to describe their strategy for expressing anger towards the target character during NF. Subjects' anger-expression strategy predominantly comprised of imagining being part of the scene and confronting the target character either verbally (e.g., rebuke and shouting), physically (e.g., pushing the target character or beating him up), or a combination of the two. These strategies were used in nearly $80 \%$ of all blocks, each subject applied them and, with the exception of a single subject with no successful blocks, everybody succeeded with them at least once. Moreover, each subject tried one of these simple strategies out first: 4 subjects applied a verbal strategy during their first block, 2 applied physical, and 5 applied a combined verbal-physical. The most successful strategy was physical only, which worked $75 \%$ of the time it was applied, followed by a combination of verbal and physical (48\% success rate) and verbal only (36\% success). This confirms that subjects were able to elicit natural cognitive strategies for anger despite minimal instructions, with a clear indication that some of the most successful ones included an approach component.

\section{Results Interpretation}

The above results confirm that when instructed to express anger, subjects show increased levels of left prefrontal asymmetry [16]. We have considered alternative explanations for the observed physiological response, but these are not supported by our experimental data. The effect of concentration alone cannot explain the observed asymmetry, as subjects rated the counting task as mentally difficult as the NF task, suggesting similar concentration levels. Wilcoxon signed-rank test revealed no statistically significant difference in subjective difficulty between Count $(\mathrm{M}=5.41, \mathrm{SD}=1.45)$ and Anger $(\mathrm{M}=6.91, \mathrm{SD}=2.26)$ tasks rated on a 10 -point scale, $\mathrm{T}=15, \mathrm{z}=-1.608, \mathrm{p}=.11$.

Negative thoughts towards the character cannot explain the observed asymmetry, as PFC asymmetry is not induced by negative valence [7]. Finally, a form of preoccupation for the victim character, known as concern empathy [24], would actually decrease, rather than increase, left PFC asymmetry [24]. Furthermore, if we consider that successful subjects reported high levels of expressed anger, and the magnitude of left asymmetry during NF was significantly correlated with STAXI-2 trait anger, we can conclude that experimental results are compatible with our initial hypothesis.
An overall success for $73 \%$ of first-time users with minimal training is consistent with the state-of-the-art in fNIRSbased BCI [19]. It should be noted that typical NF experiments (in clinical research) include extensive training spread over multiple sessions: for instance, Rosenfeld et al. [35] reported training subjects over three days prior to NF experiments, and Kotchoubey et al. [23] up to eight weeks. These results, together with accepted figures of $\mathrm{BCI}$ illiteracy of around $20 \%$, are encouraging in terms of future usability of the system. In addition, 7 subjects (64\%) succeeded within the first two blocks (4 of whom succeeded in both blocks).

\section{Perspectives for User Interface development}

The ability to control approach as an affective dimension possesses an important potential for user interaction, which is only beginning to be explored. Firstly, approach is related to motivational processes, which is an important element of a user state to capture, in particular when its value can be controlled over a continuous range. Secondly, approach naturally relates to a specific target, which can be made an explicit element of the interface layout (e.g., a virtual agent in a narrative, and a landmark or a destination in a map). The experiment we presented suggests that approach can be selectively controlled independently of valence, which is an important confirmation for its use in interactive systems. The actual use of approach in user interfaces can be subject to various design decisions depending on the nature of the application. For instance, some systems may only use approach in a positive valence context, while other may actually use anger itself (in training or entertainment applications). Systems that would rely on the use of approach in both contexts may require an additional channel capturing valence, although the context itself could also provide disambiguation.

\section{CONCLUSIONS}

We have shown that fNIRS could support the implementation of an active, affective BCI based on approach, under a NF paradigm. Subjects were able to successfully use the interface with minimal training, and BCI results were correlated with self-reported anger. Compared with previous work based on EEG prefrontal asymmetry [9], our approach harnesses the standard benefits of fNIRS $[17,29]$, in terms of spatial resolution (in particular in the prefrontal area) and greater signal stability. An advantage of our system is that it relies on relatively simple calculations and does not require complex online categorization algorithms. Our results can inform the design of affective BCI associated with prefrontal asymmetry, which is involved in a wide range of user mental states, from empathy to anger, or even risk-taking. 


\section{REFERENCES}

1. Afergan, D. Using brain-computer interfaces for implicit input. In Proceedings of the Adjunct Publication of the 27th Annual ACM Symposium on User Interface Software and Technology, UIST'14 Adjunct, ACM (New York, NY, USA, 2014), 1316.

2. Afergan, D., Peck, E. M., Solovey, E. T., Jenkins, A., Hincks, S. W., Brown, E. T., Chang, R., and Jacob, R. J. Dynamic difficulty using brain metrics of workload. In Proceedings of the SIGCHI Conference on Human Factors in Computing Systems, CHI '14, ACM (New York, NY, USA, 2014), 3797-3806.

3. Afergan, D., Shibata, T., Hincks, S. W., Peck, E. M., Yuksel, B. F., Chang, R., and Jacob, R. J. Brain-based target expansion. In Proceedings of the 27th Annual ACM Symposium on User Interface Software and Technology, UIST '14, ACM (New York, NY, USA, (2014), 583-593.

4. Ayaz, H. Functional near infrared spectroscopy based brain computer interface. $\mathrm{PhD}$ thesis, Drexel University, 2010.

5. Ayaz, H., Shewokis, P. A., Bunce, S., and Onaral, B. An optical brain computer interface for environmental control. In Engineering in Medicine and Biology Society, EMBC, 2011 Annual International Conference of the IEEE, IEEE (2011), 6327-6330.

6. Ayaz, H., Shewokis, P. A., Bunce, S., Schultheis, M., and Onaral, B. Assessment of cognitive neural correlates for a functional near infrared-based brain computer interface system. In Foundations of Augmented Cognition. Neuroergonomics and Operational Neuroscience. Springer, 2009, 699-708.

7. Berkman, E. T., \& Lieberman, M. D. Approaching the bad and avoiding the good: Lateral prefrontal cortical asymmetry distinguishes between action and valence. Journal of Cognitive Neuroscience 22, 9 (2010), 19701979.

8. Bunce, S. C., Izzetoglu, M., Izzetoglu, K., Onaral, B., and Pourrezaei, K. Functional near-infrared spectroscopy. Engineering in Medicine and Biology Magazine 25, 4 (2006), 54-62.

9. Cavazza, M., Charles, F., Aranyi, G., Porteous, J., Gilroy, S. W., Raz, G., Keynan, N. J., Cohen, A., Jackont, G., Jacob, Y., Soreq, E., Klovatch, I., and Hendler, T. Towards emotional regulation through neurofeedback. In Proceedings of the 5th Augmented Human International Conference, AH '14, ACM (New York, NY, USA, 2014), 42:1-42:8.

10. Davidson, R. J. Anterior cerebral asymmetry and the nature of emotion. Brain and cognition 20, 1 (1992), $125-151$.
11. Doi, H., Nishitani, S., and Shinohara, K. NIRS as a tool for assaying emotional function in the prefrontal cortex. Frontiers in Human Neuroscience 7 (2013), Article 770.

12. Gilroy, S. W., Porteous, J., Charles, F., Cavazza, M., Soreq, E., Raz, G., Ikar, L., Hendler, T., A braincomputer interface to a plan-based narrative. In Proceedings of the Twenty-Third international joint conference on Artificial Intelligence (IJCAI), (2013), 1997-2005.

13. Girouard, A., Solovey, E., Hirshfield, L., Chauncey, K., Sassaroli, A., Fantini, S., and Jacob, R. Distinguishing difficulty levels with non-invasive brain activity measurements. In Human-Computer Interaction INTERACT 2009, T. Gross, J. Gulliksen, P. Kotz, L. Oestreicher, P. Palanque, R. Prates, and M. Winckler, Eds., vol. 5726 of Lecture Notes in Computer Science, Springer Berlin Heidelberg (2009), 440-452.

14. Girouard, A., Solovey, E., Hirshfield, L., Peck, E., Chauncey, K., Sassaroli, A., Fantini, S., and Jacob, R. From brain signals to adaptive interfaces: Using fNIRS in HCI. In Brain-Computer Interfaces, D. S. Tan and A. Nijholt, Eds., Human-Computer Interaction Series. Springer London, 2010, 221-237.

15. Harmon-Jones, E. Clarifying the emotive functions of asymmetrical frontal cortical activity. Psychophysiology 40, 6 (2003), 838-848.

16. Harmon-Jones, E., and Sigelman, J. State anger and prefrontal brain activity: evidence that insult-related relative left-prefrontal activation is associated with experienced anger and aggression. Journal of Personality and Social Psychology 80, 5 (2001), 797803.

17. Hirshfield, L. M., Gulotta, R., Hirshfield, S., Hincks, S., Russell, M., Ward, R., Williams, T., and Jacob, R. This is your brain on interfaces: Enhancing usability testing with functional near-infrared spectroscopy. In Proceedings of the SIGCHI Conference on Human Factors in Computing Systems, CHI '11, ACM (New York, NY, USA, 2011), 373-382.

18. Hirshfield, L. M., Solovey, E. T., Girouard, A., Kebinger, J., Jacob, R. J., Sassaroli, A., and Fantini, S. Brain measurement for usability testing and adaptive interfaces: An example of uncovering syntactic workload with functional near infrared spectroscopy. In Proceedings of the SIGCHI Conference on Human Factors in Computing Systems, CHI '09, ACM (2009), 2185-2194. 
19. Hoshi, Y., Huang, J., Kohri, S., Iguchi, Y., Naya, M., Okamoto, T., and Ono, S. Recognition of human emotions from cerebral blood flow changes in the frontal region: a study with event related near-infrared spectroscopy. Journal of Neuroimaging 2, 2 (2011), 94-101.

20. Hwang, H. J., Lim, J. H., Kim, D. W., and Im, C. H. Evaluation of various mental task combinations for near-infrared spectroscopy-based brain-computer interfaces. Journal of Biomedical Optics 19, 7 (2014), 077005.

21. Karran, A. J., Fairclough, S. H., and Gilleade, K. A framework for psychophysiological classification within a cultural heritage context using interest. $A C M$ Transactions on Computer-Human Interaction (TOCHI) 21, 6 (2015), 34.

22. Kim, S., and Hamann, S. Neural correlates of positive and negative emotion regulation. Journal of Cognitive Neuroscience 19, 5 (2007), 776-798.

23. Kotchoubey, B., Kubler, A., Strehl, U., Flor, H., Birbaumer, N., Can humans perceive their brain states? Consciousness and Cognition 11, (2002), 98-113.

24. Light, S. N., Coan, J. A., Zahn-Waxler, C., Frye, C., Goldsmith, H. H., and Davidson, R. J. Empathy is associated with dynamic change in prefrontal brain electrical activity during positive emotion in children. Child Development 80, 4 (2009), 1210-1231.

25. Lotte, F., Larrue, F., and Mühl, C. Flaws in current human training protocols for spontaneous braincomputer interfaces: lessons learned from instructional design. Frontiers in Human Neuroscience 7 (2013).

26. Moghimi, S., Kushki, A., Power, S., Guerguerian, A. M., and Chau, T. Automatic detection of a prefrontal cortical response to emotionally rated music using multi-channel near-infrared spectroscopy. Journal of Neural Engineering 9, 2 (2012), 026022.

27. Morinaga, K., Akiyoshi, J., Matsushita, H., Ichioka, S., Tanaka, Y., Tsuru, J., and Hanada, H. Anticipatory anxiety-induced changes in human lateral prefrontal cortex activity. Biological Psychology 74, 1 (2007), 3438.

28. Mühl, C., Allison, B., Nijholt, A., and Chanel, G. A survey of affective brain computer interfaces: principles, state-of-the-art, and challenges. BrainComputer Interfaces 1, 2 (2014), 66-84.

29. Naseer, N., and Hong, K. fNIRS-based brain-computer interfaces: a review. Frontiers in Human Neuroscience 9, 3 (2015), Article 3.
30. Oum, K., Ayaz, H., Shewokis, P. A., and Diefenbach, P. Mindtactics: A brain computer interface gaming platform. In Games Innovations Conference (ICEGIC), 2010 International IEEE Consumer Electronics Society's, IEEE (2010), 1-5.

31. Peck, E. M., Afergan, D., and Jacob, R. J. K. Investigation of fNIRS brain sensing as input to information filtering systems. In Proceedings of the 4th Augmented Human International Conference, $\mathrm{AH}$ '13, ACM (2013), 142-149.

32. Peck, E. M. M., Yuksel, B. F., Ottley, A., Jacob, R. J., and Chang, R. Using fNIRS Brain Sensing to Evaluate Information Visualization Interfaces. In Proceedings of the SIGCHI Conference on Human Factors in Computing Systems, CHI '13, ACM (2013), 473-482.

33. Pike, M. F., Maior, H. A., Porcheron, M., Sharples, S. C., and Wilson, M. L. Measuring the effect of think aloud protocols on workload using fNIRS. In Proceedings of the SIGCHI Conference on Human Factors in Computing Systems, ACM (2014), 38073816.

34. Poole, B. D., and Gable, P. A. Affective motivational direction drives asymmetric frontal hemisphere activation. Experimental Brain Research 232, 7 (2014), 2121-2130.

35. Rosenfeld, J. P., Cha, G., Blair, T., and Gotlib, I. H. Operant (biofeedback) control of left-right frontal alpha power differences: Potential neurotherapy for affective disorders. Biofeedback and Self-Regulation 20, 3 (1995), 241-258.

36. Ruocco, A. C., Rodrigo, A. H., Lam, J., Di Domenico, S. I., Graves, B., and Ayaz, H. A problem-solving task specialized for functional neuroimaging: validation of the Scarborough adaptation of the Tower of London (S-TOL) using near-infrared spectroscopy. Frontiers in Human Neuroscience 8 (2014), Article 185.

37. Sakatani, K., Takemoto, N., Tsujii, T., Yanagisawa, K., and Tsunashima, H. NIRS-Based Neurofeedback Learning Systems for Controlling Activity of the Prefrontal Cortex. In Oxygen Transport to Tissue $X X X V$, S. Van Huffel, G. Naulaers, A. Caicedo, D. F. Bruley, and D. K. Harrison, Eds., vol. 789 of Advances in Experimental Medicine and Biology. Springer New York (2013), 449-454.

38. Sarkheil, P., Zilverstand, A., Kilian-Htten, N., Schneider, F., Goebel, R., and Mathiak, K. fMRI feedback enhances emotion regulation as evidenced by a reduced amygdala response. Behavioural Brain Research 281 (2015), 326-332. 
39. Solovey, E. T., Girouard, A., Chauncey, K., Hirshfield, L. M., Sassaroli, A., Zheng, F., Fantini, S., and Jacob, R. J. Using fNIRS Brain Sensing in Realistic HCI Settings: Experiments and Guidelines. In Proceedings of the 22nd Annual ACM Symposium on User Interface Software and Technology, UIST '09, ACM (2009), 157-166.

40. Solovey, E. T., Okerlund, J., Hoef, C., Davis, J., and Shaer, O. Augmenting spatial skills with semiimmersive interactive desktop displays: Do immersion cues matter? In Proceedings of the 6th Augmented Human International Conference, AH '15, ACM (2015), 53-60.

41. Spielberger, C. D. Staxi-2: state-trait anger expression inventory-2; professional manual, 1999.

42. Tai, K., and Chau, T. Single-trial classification of NIRS signals during emotional induction tasks: towards a corporeal machine interface. Journal of NeuroEngineering and Rehabilitation 6 (2009), 39.
43. Tuscan, L.-A., Herbert, J. D., Forman, E. M., Juarascio, A. S., Izzetoglu, M., and Schultheis, M. Exploring frontal asymmetry using functional near-infrared spectroscopy: a preliminary study of the effects of social anxiety during interaction and performance tasks. Brain Imaging and Behavior 7, 2 (2013), 140153.

44. Wehbe, R. R., Kappen, D. L., Rojas, D., Klauser, M., Kapralos, B., and Nacke, L. E. EEG-based Assessment of Video and In-game Learning. In CHI '13 Extended Abstracts on Human Factors in Computing Systems, CHI EA '13, ACM (2013), 667-672.

45. Zotev, V., Phillips, R., Yuan, H., Misaki, M., and Bodurka, J. Self-regulation of human brain activity using simultaneous real-time fMRI and EEG neurofeedback. NeuroImage 85 (2014), 985-995. 